\title{
Application of concrete deformation model for calculation of bearing capacity of reinforced concrete structures
}

\author{
Vasiliy Murashkin ${ }^{1}$, and Gennadiy Murashkin ${ }^{2, *}$ \\ ${ }^{1}$ Limited Liability company KB "Kuibyshev project" \\ ${ }^{2}$ Samara State Technical University, Academy of Architecture and Civil Engineering, \\ Molodogvardeyskaya St., 194, Samara, 443001, Russia
}

\begin{abstract}
The paper presents a brief historical reference of the theory of reinforced concrete structures calculation and its. It shows that modeling of concrete deformations makes it possible not only to carry out calculations of durability and to determine reinforced concrete elements deflection under the estimated load, but also to analyze their a stress-strain state at each stage. The study suggests a new model of concrete deformation which makes it possible to make calculations of concrete elements with characteristics which are different from those given in specification documents. The researchers use the proposed model of deformation and calculate curved reinforced concrete beam bearing capacity. These calculations for normally reinforced elements do not considerably differ from the calculations given in the documents. All the main functional dependencies on the calculation stages are visualized.
\end{abstract}

\section{Introduction}

Reinforced concrete is a relatively new structural material. When it first appeared, the structural mechanics methodology (which in itself is calculations according to working stresses) was adapted for reinforced concrete structures. This was the first stage of the theory of reinforced concrete calculations. At the 2nd All-Union Conference on concrete and reinforced concrete in 1932, Arthur F. Loleit, a professor of the Department of Military Engineering Academy, made a report on a fundamentally new approach to the determination of the strength of reinforced concrete structures on the basis of destructive load calculations. It was the world's first original solution of the problem of physical nonlinear nature of concrete and steel at their combined action. During his experimental data analysis, Arthur Loleit found out that the destruction of a bending reinforced concrete construction is a process which is long enough. It usually begins with the opening of cracks and the yeild of concrete reinforcement. At the same time, the initial height of the compressed zone decreases, and the stresses in the compressed zone of concrete grow and reach the limit values even at the yield of reinforcement. It is typical to most bending

\footnotetext{
Corresponding author: murvag@mail.ru
} 
elements. Later, they were called "normally reinforced". Professor Loleit's report at the conference were followed by a heated discussion. Such famous scientists in the field of building construction as Vsevolod M. Keldysh, Yakov V. Stolyarov, Boris G. Skramtaev, Viktor P. Nekrasov never understand the validity of the stated method.

In two years, Aleksey A. Gvozdev carried out special experiments and proved that Arthur Loleit's calculations were exceptionally accurate, dis not require technical knowledge of the deformation curve of concrete, and at the same time showed a more accurate picture of durability compared to the previous methods of calculating reinforced concrete bending structures. It was the second stage of the development of reinforced concrete theory. Many scientists who did not previously support Arthur Loleit theory changed their attitude after the Aleksey Gvozdev's report. It has allowed to create essentially new calculation specifications of reinforced concrete structures. It was a breakthrough in the world's practice which put out Russian scientists ahead of foreign builders for many decades. Later, due to the Russian scientists, a method of calculation according limit states was firstly introduced. It was based on the "Loleit's principle". Russian scientists were the pioneers of applying calculations of reinforced concrete structures according to destructive loads. Later, they were the first to calculate deflections of structures according to their limit states. It was another breakthrough, this time in understanding the influence of plastic deformations on the work of the structure at the moment of destruction. Up to the present moment all engineers use the achievements of Russian scientists in calculations of constructions on the basis of their limit states.

However, the calculations according to limit states do not solve all the problems. Thus, they do not explain the condition of the construction under the operational load, the change of strength and deformation characteristics of concrete in time, the overhaul life of construction, etc. That is why the research of nonlinear concrete deformation processes continues. It tries to find more adequate to experiment, but at the same time simpler models of deformation of materials $[1,8,10]$.

\section{Research Methods}

\subsection{Description of the concrete deformation model}

The third stage of development of the theory of reinforced concrete started when models of concrete deformation were introduced both in Russian specification documents and foreign normative documents [4-11].

Nikolay I. Karpenko, a member of Russian Academy of Architecture and Construction Sciences, developed a model showing dependence of deformations on tensions $\varepsilon=f(\sigma)$. It was based on the statistical analysis of numerous experiments. This model corrected all received earlier models, including those given in other countries specifications. Specifications SP 63. 13330.2012 recommend using Nikolay Karpenko's model in reinforced concrete calculations $[2,3]$. The model is designed to be used for concrete, which characteristics meet specifications regulations. Therefore, this model is used in such program complexes (PC) for structures calculation as PC Lira, SKAD, etc.

The model of deformation proposed in this work is based on the dependence reverse to the model described in Paper [3] $-\sigma=f(\varepsilon)$.

The model presented in Paper [1] is defined for concrete on the basis of the obtained values of concrete strength, modulus of elasticity and deformation at the peak of stresses. All these characteristics are not connected to each other and can be obtained on the basis of standard tests carried out during structures manufacture or obtained from working 
structures inspection results. The personified model almost coincides with the model given in Paper [3] according to characteristics of concrete standards which correspond to "standard" norms. At the same time, the proposed model can also be used for "non-standard concrete", such as plastoconcrete, concrete hardening under pressure, various fibreconcretes and other concrete types in which deformation characteristics do not correspond to the existing standards. The positive aspect of the model proposed in Paper [1] is its relative simplicity of solutions visualization together with the use of standard applications of mathematical programs to the $\mathrm{PC}$, which do not require special programs development. At the moment, there is no technological gap and the application of computing technology between Russia and the other world. In some cases, scientific developments of Russian scientists are even more important than those of western researchers. Therefore, the use of new achievements in research of nonlinear nature of concrete deformation and their use for practical purposes allow to create most modern reinforced concrete constructions.

Paper [2] gives Example 45 of calculating deflections of reinforced concrete slab freely supported on the ends with dimensions of $\mathrm{h}=20 \mathrm{~cm}, \mathrm{~b}=100 \mathrm{~cm}, 1=560 \mathrm{~cm}$, made of concrete with $\mathrm{Rb}=110 \mathrm{~kg} / \mathrm{cm} 2, \mathrm{~Eb}=240000 \mathrm{~kg} / \mathrm{cm} 2$, and working reinforcement in the lower flange As $=7.69 \mathrm{~cm} 2(5 \varnothing 1.4 \mathrm{~cm}), \mathrm{h} 0=17.3 \mathrm{~cm}$ (see Page 171).

According to the Russian standards (see Paper [3]), the concrete slab load capacity from Example 45 is:

$$
\xi<\xi r \quad M_{\text {norm }}=R s * A s *\left(h_{0}-x c n / 2\right)=4.895 * 10^{5}
$$

\subsection{Mathematical model}

The mathematical model of concrete nonlinear deformation proposed in Paper [1] is as follows (2):

$$
\sigma b(\varepsilon b)=a^{*} \varepsilon b^{b * \exp \left(-b^{*} \varepsilon b / p\right)}
$$

where: $a=4.16^{*} 10^{4} ; b=0.823 ; p=2 * 10^{-3}$.

Values $\mathrm{a}$ and $\mathrm{b}$ are derived from the solution of equations that are based on the following: 1 - on the assumption that concrete works elastic up to stresses that are equal $10 \%$ from durability, 2 - on the assumption that stresses maximum is observed at deformations $\mathrm{p}$ in concrete. Deformations $\mathrm{p}$ are determined by equation G-8 given in Paper [3]. Figure 1 shows a graphic image of the proposed concrete deformation model.

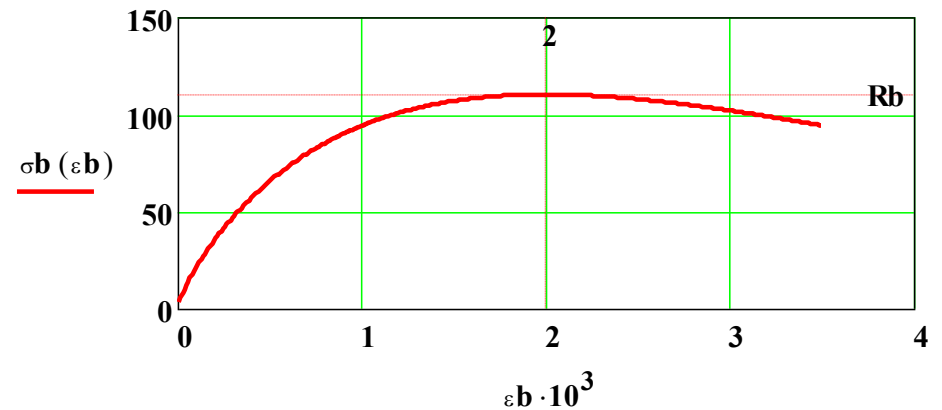

Fig. 1. Graph of the proposed model of nonlinear deformation of concrete. 


\section{Results}

The height of the compressed concrete zone $-\mathrm{L}(\varepsilon b)$ depending on $\varepsilon b$ is determined from the conditions of equality of efforts in the stretched and compressed zones.

$$
N s(\varepsilon b)-D(\varepsilon b)=0
$$

Resultant forces in compressed concrete are determined from the condition (3):

$$
D(\varepsilon b)=\int_{0}^{L(\varepsilon b)} \sigma b\left(\frac{\varepsilon b}{L(\varepsilon b)} * x\right) * b d x
$$

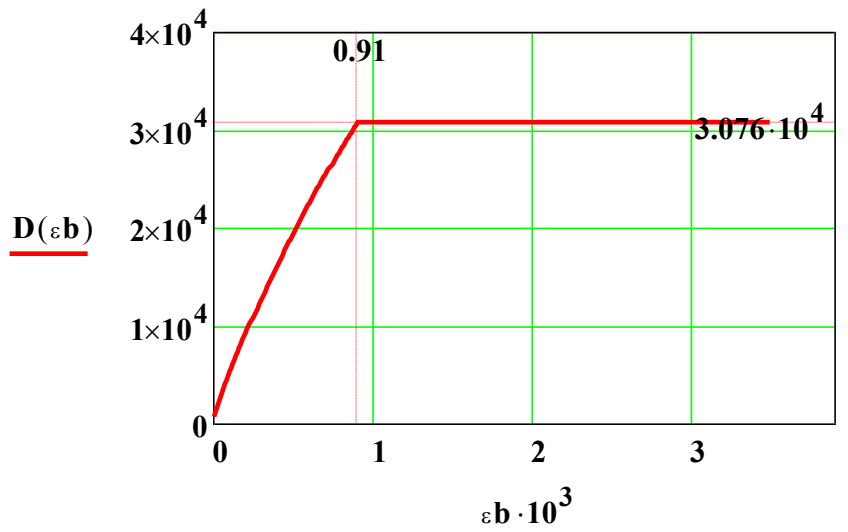

Fig. 2. Schedule of forces in the compressed area of concrete.

Stresses in the stretched reinforcement are determined by the formula (4):

$$
\sigma s(\varepsilon b)=\frac{\varepsilon b}{L(\varepsilon b)} *(h 0-L(\varepsilon b)) * E s \text { if } \quad \frac{\varepsilon b}{L(\varepsilon b)} *(h 0-L(\varepsilon b)) * E s \geq R s
$$

Rs otherwise.

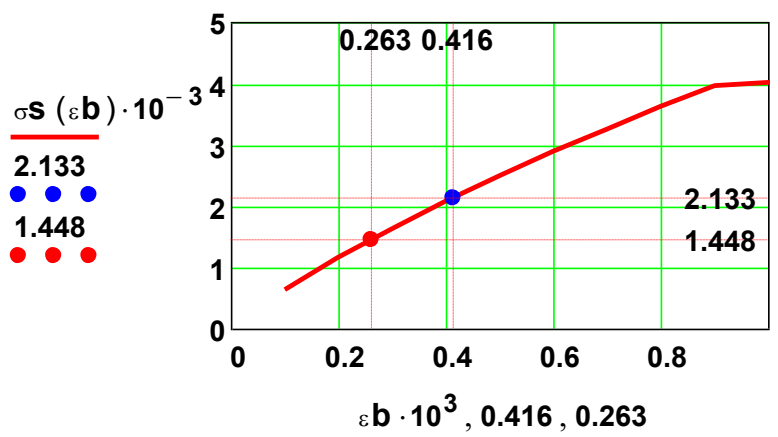

Fig. 3. Graphical representation of stresses in a stretched armature. The points show the level of crack formation and design load.

Force in reinforcement (excluding stretched concrete) is equal to $\mathrm{Ns}(\varepsilon b)=\sigma s(\varepsilon b)^{*} \mathrm{As}$. 


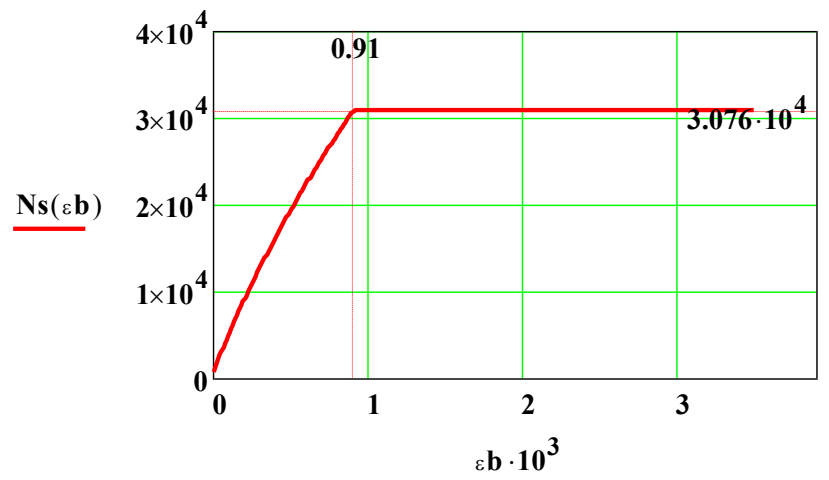

Fig. 4. A graphical representation of effort in the stretched armature.

The height of the compressed zone is determined from the expression (5):

$$
L(\varepsilon b)=\operatorname{root}\left[\frac{\int_{0}^{k} \sigma b\left(\frac{\varepsilon b}{k} * x\right) * b d x}{\frac{\varepsilon b}{k} *(h 0-k) * E s^{*} A s}-1, k\right]
$$

$$
\text { if } \frac{\varepsilon b}{k} *(h 0-k) * E s \leq R s \quad(R s * A s) \quad \text { otherwise }
$$

Though there are numerous models of concrete nonlinear deformation given in literary sources, there is no mathematical expression for height of the compressed zone and its graphic representation which makes it possible to exclude deflections calculations and use the approach of classical building mechanics.

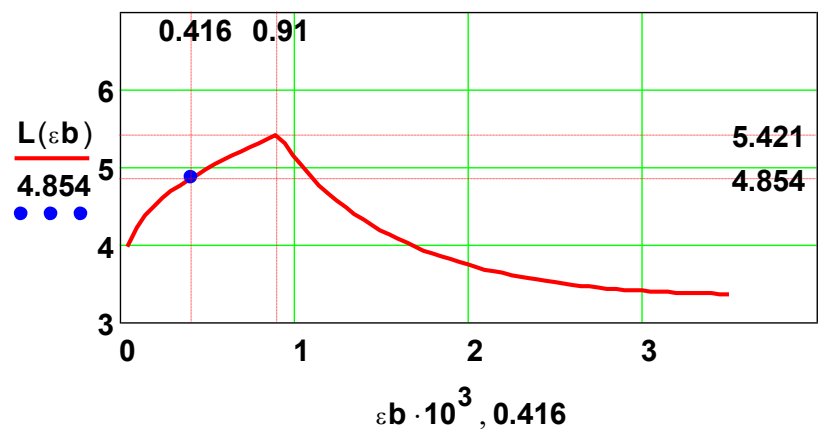

Fig. 5. Graph of function L( $(\varepsilon b)$.

Figure 5 demonstrates that the fracture of the curve occurs when the reinforcement reaches its yield limit at $\varepsilon b=0.00091$. The point on the curve is the value of the compressed zone height at the calculated load Mcal $=2.548 * 105 \mathrm{~kg} / \mathrm{cm}$ for calculations of deflections of a plate given in Example 45 of Paper [2].

Let us define the shoulder of the inner pair of forces by the expression (6): 


$$
Z(\varepsilon b)=\frac{\int_{0}^{L(\varepsilon b)} \sigma b\left(\frac{\varepsilon b}{L(\varepsilon b} * x\right) * b * x d x}{\int_{0}^{L(\varepsilon b)} \sigma b\left(\frac{\varepsilon b}{L(\varepsilon b)}\right) * b d x}+(h 0-L(\varepsilon b))
$$

The graphic image of the dependence of the inner pair of forces on deformations is shown in Figure 6.

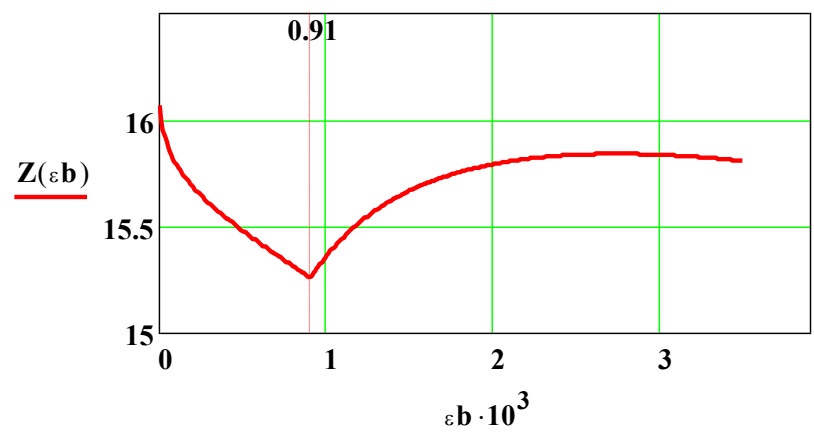

Fig. 6. Graph of function $Z(\varepsilon b)$.

Let's define the dependence of the current moment as the function $\varepsilon b(7)$ :

$$
M(\varepsilon b)=Z(\varepsilon b) * D(\varepsilon b)
$$

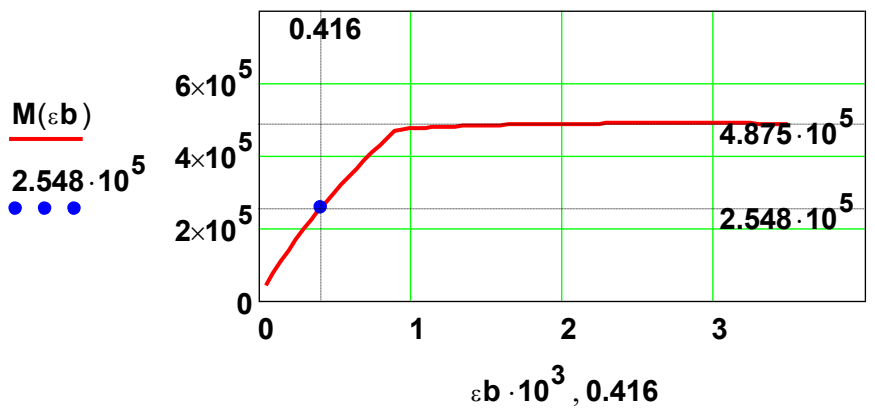

Fig. 7. Graph of function $M(\varepsilon b)$.

At load equal to calculated Mcal $=2,548 * 10^{5} \mathrm{~kg} * \mathrm{~cm}$, deformation of the concrete compressed zone makes $\varepsilon b=0.000416$.

At deformation of the top fibre of the compressed concrete $\varepsilon b=0.0028$, we get the value of the maximum moment: $M \bmod =M\left(2.8 * 10^{-3}\right)=4.875 * 10^{5}$.

The comparison of Mmod $=4.875 * 10^{5} \mathrm{~kg}^{*} \mathrm{~cm}$ with Mnorm $=4.895 * 10^{5} \mathrm{~kg} * \mathrm{~cm}$ shows that calculations of bending reinforced concrete elements with application of the proposed model of deformation are correct. But unlike the existing specifications, the use of the deformation model allows to get stresses and deformations at any level of structural load, as well as more accurately calculate structural deflections. 


\section{Discussion}

Russian normative documents specifying calculations of reinforced concrete structures [3] and in norms of the foreign countries [4] recommend the use of deformation models in which durability of concrete is connected with modulus of elasticity. This connection is typical for "standard" concretes used in designing new structures. Application of the proposed model of nonlinear concrete deformation for concrete structures which are made of concrete with characteristics corresponding to normative values given in Papers [3.4] does not lead to significant discrepancy of calculation results. At the same time, with longterm operation of the building, there may be a violation of the connection between the deformational characteristics of concrete and its strength given in specification documents. In this case, the use of normative models can give incorrect calculation results.

The proposed model of concrete nonlinear deformation is formed on the basis of experimental data and normative specifications with practically any combinations of concrete durability and its modulus of elasticity. Therefore, it is possible to use the model both for long-time structures and for new types of concrete developed by researchers.

\section{Conclusions}

The model of nonlinear concrete deformation proposed in the paper is more general in comparison with models given normative specification. For concretes, corresponding to the normative values according all indicators, the proposed model provides convergence with calculations according to limit states. For calculation of long-term operating constructions and constructions from new types of concretes, it is necessary to conduct experimental research and reveal actual concrete durability, modulus of elasticity and limiting deformability.

\section{References}

1. G.V. Murashkin, V.G. Murashkin, Higher Educ. Estab. News, Constr., 10, 4-6 (1997)

2. SP 52-101-2003, Manual for the design of concrete structures made of heavy concrete without pre-stress reinforcement (Moscow, 2003)

3. SP 63.133302.2012, Concrete and concrete structures: Fundamental principles (Moscow, 2012)

4. Part 1.1 prEN 1992-1, Eurocode 2: Design of concrete structures (1992)

5. N.I. Karpenko, General models of reinforced concrete mechanics (Moscow, 1996)

6. G.V. Murashkin, V.G. Murashkin, Fund. Research on Sc. Devel. of Arch. and Costr. Indust., 1, 535-543 (2016)

7. V.V. Petrov, Non-linear incremental construction mechanics (Moscow, 2014)

8. E.N. Kodysh, N.N. Trekin, I.K. Nikitin, K.E. Sosedov, Practical methods and examples of calculation of reinforced concrete structures from heavy concrete by SP 63.13330 (Moscow, 2017)

9. V.G. Kazachek, N.V. Nechaev, S.N. Notenko, V.I. Rimshin, A.G. Roitman, Inspection and testing of buildings and structures (Moscow, 2004)

10. V.M. Bondarenko, V.I. Kolchunov, Calculated models of reinforced concrete resistance (Moscow, 2004)

11. V.O. Almazov, Designing reinforced concrete structures by Evronorms (Moscow, 2011) 